\title{
Coding Along Hermite Polynomials for Interference Channels
}

\author{
Emmanuel A. Abbe \\ LCM, EPFL \\ Lausanne, $1015 \mathrm{CH}$ \\ Email: eabbe@mit.edu
}

\author{
Lizhong Zheng \\ RLE, LIDS, MIT \\ Cambridge, MA 02139 \\ Email: lizhong@mit.edu
}

\begin{abstract}
This paper analyzes the use of non-Gaussian input distributions over the Gaussian interference channel. It has been recently proved that the iid Gaussian code ensemble together with a decoder that treats interference as noise is sum-capacity achieving, if the interference is below a threshold. We show that, when the decoder treats interference as noise, and when the interference is above a threshold, the iid Gaussian ensemble can be strictly improved upon. In the block synchronous setting, the improvement is obtained by a Gaussian but non iid ensemble, whereas in the asynchronous setting, it is obtained by an iid but non Gaussian ensemble. The analysis of non-Gaussian ensembles is made possible by the use of the Hermite coordinate system.
\end{abstract}

\section{INTRODUCTION}

Let a memoryless additive white Gaussian noise (AWGN) channel be described by $Y=X+Z$, where $Z \sim \mathcal{N}(0, v)$ is independent of $X$. If the input is subject to an average power constraint given by $\mathbb{E} X^{2} \leq p$, the input distribution maximizing the mutual information is Gaussian. This is due to the fact that under second moment constraint, the Gaussian distribution maximizes the entropy, hence

$$
\arg \max _{X: \mathbb{E} X^{2}=p} h(X+Z) \sim \mathcal{N}(0, p) .
$$

On the other hand, for an additive noise channel, if we use a Gaussian input distribution, i.e., $X \sim \mathcal{N}(0, p)$, among noises with bounded second moment, the noise minimizing the mutual information is again Gaussian. This can be shown by using the entropy power inequality (EPI), which reduces in this setting to $\arg \min _{Z: h(Z)=\frac{1}{2} \log 2 \pi e v} h(X+Z) \sim \mathcal{N}(0, v)$ and implies

$$
\arg \min _{Z: \mathbb{E} Z^{2}=v} h(X+Z)-h(Z) \sim \mathcal{N}(0, v) .
$$

Hence, in the single-user setting, Gaussian inputs are the best inputs to fight Gaussian noise and Gaussian noise is the worst noise to face with Gaussian inputs. This provides a game equilibrium between user and nature, as defined in [5], p. 263. With these results, many problems in information theory dealing with Gaussian noise can be solved. However, in the network information theory setting, two interesting new phenomena make the search for the optimal input distributions more complex. First, the interference. The fact that there are other users in the system brings an interesting "frustration phenomena". Since the multiple users produce interference on each other, it is no longer clear that Gaussian inputs are the best input distributions for the overall system. Indeed, let us assume that there are two users and that the first one decides to use iid Gaussian inputs. Then the second user should use Gaussian inputs to maximize his own rate, but this would also cause the most harmful interference on the first user, if he treats interference as noise. How to treat the interference and how to optimally construct codes on such channels is still an open problem. Then comes the fading. Over a single-user AWGN channel with coherent fading, it is clear that maximizing $I(X ; Y \mid H)$ is achieved by a Gaussian input, according to (1). However, even for the broadcast AWGN channel with coherent fading, it has been an open problem whether Gaussian inputs are still optimal or not. A reason for these open questions, is that Gaussian inputs are pretty much the only ones that can be analyzed over Gaussian noise channels, as non-Gaussian inputs leave most problems in an intractable form. In [1], a novel technique has been developed to analyze a class of non-Gaussian input distributions over Gaussian noise channels. It allowed in particular to find nonGaussian input distributions that outperform Gaussian ones over the broadcast AWGN channel with coherent fading. In this paper, we focus on the other phenomena mentioned above, namely, the interference.

\section{Problem Statement}

We consider the symmetric memoryless interference channel (IC) with two users and white Gaussian noise. The average power is denoted by $p$, the interference coefficients by $a$, and the respective noise by $Z_{1}$ and $Z_{2}$ (independent standard Gaussian). We define the following expression

$$
\begin{aligned}
& n S_{a, p}\left(X_{1}^{n}, X_{2}^{n}\right) \\
& =I\left(X_{1}^{n} ; X_{1}^{n}+a X_{2}^{n}+Z_{1}^{n}\right)+I\left(X_{2}^{n} ; X_{2}^{n}+a X_{1}^{n}+Z_{2}^{n}\right) \\
& =h\left(X_{1}^{n}+a X_{2}^{n}+Z_{1}^{n}\right)-h\left(a X_{2}^{n}+Z_{1}^{n}\right) \\
& \quad+h\left(X_{2}^{n}+a X_{1}^{n}+Z_{2}^{n}\right)-h\left(a X_{1}^{n}+Z_{2}^{n}\right)
\end{aligned}
$$

where $X_{1}^{n}$ and $X_{2}^{n}$ are independent random vectors with a covariance having a trace bounded by $n p$ and $Z_{i}^{k}, i=1,2$, $k=1, \ldots, n$, are iid standard Gaussian. For any dimension $n$ and any distributions of $X_{1}^{n}$ and $X_{2}^{n}, S_{a, p}\left(X_{1}^{n}, X_{2}^{n}\right)$ is a lower bound to the sum-capacity. Moreover, it is tight by taking $n$ arbitrarily large and $X_{1}^{n}$ and $X_{2}^{n}$ maximizing (3). 
For each user, we say that the decoder is treating interference as noise, if it does not require the knowledge of the other user's code book. However, we allow such decoders to have the knowledge of a distribution, under which the other user's code book may be drawn. This is for example necessary to construct a sum-capacity achieving code ${ }^{1}$ in [2], [6], [7], where the decoder of each user treats interference as noise but uses the fact that the other user's code book is drawn from an iid Gaussian distribution. But, if we allow this distribution to be of arbitrarily large dimension in our definition of treating interference as noise, we can get a misleading definition. Indeed, if we take $n$ large and a distribution of $X_{1}^{n}$ and $X_{2}^{n}$ maximizing (3), we can achieve rates arbitrarily close to the sum-capacity, yet, formally treating interference as noise. The problem is that the maximizing distributions in (3) may not be iid for arbitrary $n$, and knowing it at the receiver can be as much information as knowing the other user's code book (for example, if the distribution is the uniform distribution over a code book of small error probability). Hence, one has to be careful when taking $n$ large. It is indeed an interesting problem to discuss what kind of $n$-dimensional distributions would capture the meaning of treating interference as noise that we want. This points out that studying the maximizers of (3) relates to studying the concept of treating interference as noise or information. In this paper, we will only work with situations that are not ambiguous with respect to our definition of treating interference as noise.

Now, to maximize (3), a competitive situation takes place: Gaussian inputs maximize each entropy term, but we have different signs in front of these entropy terms. It has been proved recently in [2], [6], [7], that the sum-capacity is achieved by treating interference as noise and with iid Gaussian inputs, as long as $p a^{3}+a-1 / 2 \leq 0$. Hence, in this regime, the iid Gaussian distribution maximizes (3) for any $n$. On the other hand, if $a$ gets larger and larger, there should be a moment for which treating interference as noise with iid Gaussian inputs becomes penalizing (we know this happens if $a \geq 1$ ). Now, if for a range of $a$, the iid Gaussian distribution does not maximize (3), it means that iid Gaussian inputs and treating interference as noise is not capacity achieving in this range. We know that this can happen only if $p a^{3}+a-1 / 2>0$. We wonder if, when and how this happens.

We then distinguish the implication of such a threshold in both the synchronized and asynchronized users setting, as there will be an interesting distinction between these two cases. We recall how the synch and asynch settings are defined here. In the synch setting, each user of the IC sends their code word of a common block length $n$ simultaneously, i.e., at time 1, they both send the first component of their code word, at time 2 the second component, etc. In the asynch setting, each user is still using code words of the same block length $n$, however, there might be a shift between the time at which the first and second users start sending their code words. We denote this shift by $\tau$, and assume w.l.o.g. that $0 \leq \tau \leq n$. In the totally

\footnotetext{
${ }^{1}$ in a low interference regime
}

asynch setting, we assume that $\tau$ is drawn uniformly at random within $\{0, \ldots, n\}$. We may also distinguish the cases where $\tau$ is not known at the transmitter but at the receiver, and when $\tau$ is not known at both. Note that if iid inputs are used and interference is treated as noise, whether the users are synch or asynch is not affecting the rate achievability ${ }^{2}$. However, if the users want to time-share over the channel uses, such as to fully avoid their interference, they will need synchronization.

Definition 1: Time sharing over a block length $n$ (assumed to be even) with Gaussian inputs refers to using $X_{1}$ Gaussian with covariance $2 P \hat{I}_{n / 2}$ and $X_{2}$ Gaussian with covariance $2 P \hat{I}_{n / 2}^{c}$, where $\hat{I}_{n / 2}$ is a diagonal matrix with $n / 21$ 's and 0 's, and $\hat{I}_{n / 2}^{c}$ flips the 1's and 0's on the diagonal.

We will see in section IV that a blind time-sharing which allows to partially avoid interference can still be achieved for the totally asynch IC.

\section{Local Geometry and Hermite coordinates}

Let $g_{p}$ denote the Gaussian density with zero mean and variance $p$. We consider the local geometry by looking at densities of the form

$$
f_{\varepsilon}(x)=g_{p}(x)(1+\varepsilon L(x)), \quad x \in \mathbb{R}
$$

where $L: \mathbb{R} \rightarrow \mathbb{R}$ satisfies

$$
\begin{aligned}
& \inf _{x \in \mathbb{R}} L(x)>-\infty \\
& \int_{\mathbb{R}} L(x) g_{p}(x) d x=0 .
\end{aligned}
$$

Hence, with these two constraints, $f_{\varepsilon}$ is a valid density for $\varepsilon$ sufficiently small. It is a perturbed Gaussian density, in a "direction" $L$. Observe that the second moment of $f_{\varepsilon}$ equals $p$, i.e., $m_{2}\left(f_{\varepsilon}\right)=p$ iff $M_{2}(L)=\int_{\mathbb{R}} x^{2} L(x) g_{p}(x) d x=0$. We are now interested in analyzing how these perturbations affects the output through an AWGN channel. Note that, if the input is a Gaussian $g_{p}$ perturbed in the direction $L$, the output is a Gaussian $g_{p+v}$ perturbed in the direction $\frac{g_{p} L \star g_{v}}{g_{p+v}}$, since $f_{\varepsilon} \star g_{v}=g_{p+v}\left(1+\varepsilon \frac{g_{p} L \star g_{v}}{g_{p+v}}\right)$.

Convention: $g_{p} L \star g_{v}$ refers to $\left(g_{p} L\right) \star g_{v}$, i.e., the multiplicative operator precedes the convolution one.

For simplicity, let us assume in the following that the function $L$ is a polynomial satisfying (5), (6).

Lemma 1: We have $D\left(f_{\varepsilon} \| g_{p}\right)=\frac{1}{2} \varepsilon^{2}\|L\|_{g_{p}}^{2}+o\left(\varepsilon^{2}\right)$, where $\|L\|_{g_{p}}^{2}=\int_{\mathbb{R}} L^{2}(x) g_{p}(x) d x$.

Moreover, note that for any density $f$, if its first and second moments are $m_{1}(f)=a$ and $m_{2}(f)=p+a^{2}$, we have

$$
h(f)=h\left(g_{a, p}\right)-D\left(f \| g_{a, p}\right),
$$

\footnotetext{
${ }^{2}$ hence, (3) with an iid distribution for $X_{1}$ and $X_{2}$ can still be achieved for the totally asynch IC
} 
where $g_{a, p}(x)=g_{p}(x-a)$. Hence, the extremal entropic results of (1) and (2) are locally expressed as

$$
\begin{aligned}
& \arg \min _{L: M_{2}(L)=0}\left\|\frac{g_{p} L \star g_{v}}{g_{p+v}}\right\|_{g_{p+v}}^{2}=0 \\
& \arg \max _{L: M_{2}(L)=0}\left\|\frac{g_{p} L \star g_{v}}{g_{p+v}}\right\|_{g_{p+v}}^{2}-\|L\|_{g_{p}}^{2}=0,
\end{aligned}
$$

where 0 denotes here the zero function. If (8) is obvious, (9) requires a non-trivial proof. Now, if we want to make headway on the competitive situations presented in the introduction, we need more refined results than the ones above. Let us define the following mapping,

$$
\Gamma^{(+)}: L \in L_{2}\left(g_{p}\right) \mapsto \frac{g_{p} L \star g_{v}}{g_{p+v}} \in L_{2}\left(g_{p+v} ; \mathbb{R}\right),
$$

where $L_{2}\left(g_{p}\right)$ denotes the space of real functions having a finite $\|\cdot\|_{g_{p}}$ norm. This linear mapping gives, for a given perturbed direction $L$ of a Gaussian input $g_{p}$, the resulting perturbed direction of the output through additive Gaussian noise $g_{v}$. The norm of each direction in their respective spaces, i.e., $L_{2}\left(g_{p}\right)$ and $L_{2}\left(g_{p+v}\right)$, gives how far from the Gaussian distribution these perturbations are. Note that if $L$ satisfies (5)-(6), so does $\Gamma^{(+)} L$ for the measure $g_{p+v}$. The result in (9) (worst noise case) tells us that this mapping is a contraction, but for our goal, what would be helpful is a spectral analysis of this operator, to allow more quantitative results than the extreme-case results of (8) and (9). In order to do so, one can express $\Gamma^{(+)}$as an operator defined and valued in the same space, namely $L_{2}$ with the Lebesgue measure $\lambda$, which is done by inserting the Gaussian measure in the operator argument. We then proceed to a singular function/value analysis. Formally, let $K=L \sqrt{g_{p}}$, which gives $\|K\|_{\lambda}=\|L\|_{g_{p}}$, and let

$$
\Lambda: K \in L_{2}(\lambda ; \mathbb{R}) \mapsto \frac{\sqrt{g_{p}} K \star g_{v}}{\sqrt{g_{p+v}}} \in L_{2}(\lambda ; \mathbb{R})
$$

which gives $\left\|\Gamma^{(+)} L\right\|_{g_{p+v}}=\|\Lambda K\|_{\lambda}$. Denoting by $\Lambda^{t}$ the adjoint operator of $\Lambda$, we have the following.

Proposition 1: We have that $\Lambda^{t} \Lambda K=\gamma K$ holds for each pair $(K, \gamma) \in\left\{\left(\sqrt{g_{p}} H_{k}^{[p]},\left(\frac{p}{p+v}\right)^{k}\right)\right\}_{k \geq 0}$, where $H_{k}^{[p]}(x)=$ $\frac{1}{\sqrt{k !}} H_{k}(x / \sqrt{p})$ and

$$
H_{k}(x)=(-1)^{k} e^{x^{2} / 2} \frac{d^{k}}{d x^{k}} e^{-x^{2} / 2}, \quad \forall k \geq 0, x \in \mathbb{R} .
$$

The polynomials $H_{k}^{[p]}$ are the normalized Hermite polynomials (for a Gaussian distribution having variance $p$ ) and $\sqrt{g_{p}} H_{k}^{[p]}$ are called the Hermite functions. For any $p>0,\left\{H_{k}^{[p]}\right\}_{k \geq 0}$ is an orthonormal basis of $L_{2}\left(g_{p}\right)$, this can be found in [4]. Moreover, one can check that $H_{1}$, respectively $H_{2}$ perturb (as approximations but not exactly) a Gaussian distribution into another Gaussian distribution, with a different first moment, respectively second moment. For $k \geq 3$, the $H_{k}$ perturbations are not modifying the first two moments and are moving away from Gaussian distributions. However, it is formally only for even values of $k$ that (5) is verified (this will be further discussed). The following result is a version of Proposition 1 with the Gaussian measures.

\section{Proposition 2:}

$$
\begin{aligned}
& \Gamma^{(+)} H_{k}^{[p]}=\frac{g_{p} H_{k}^{[p]} \star g_{v}}{g_{p+v}}=\left(\frac{p}{p+v}\right)^{k / 2} H_{k}^{[p+v]}, \\
& \Gamma^{(-)} H_{k}^{[p+v]}:=H_{k}^{[p+v]} \star g_{v}=\left(\frac{p}{p+v}\right)^{k / 2} H_{k}^{[p]} .
\end{aligned}
$$

Last proposition implies proposition 1 , since $\Gamma^{(-)} \Gamma^{(+)} L=$ $\gamma L \Longleftrightarrow \Lambda^{t} \Lambda K=\gamma K$ for $K=L \sqrt{g_{p}}$.

Comment: these properties of Hermite polynomials and Gaussian measures are likely to be already known, in a different context or with different notations. However, what is particularly interesting here, are not only these properties by themselves, but mainly the fact that they are precisely emerging from our information theoretic setting and are helpful to solve our problems.

Summary: In words, we just saw that $H_{k}$ is an eigenfunction of the input/output perturbation operator $\Gamma^{(+)}$, in the sense that $\Gamma^{(+)} H_{k}^{[p]}=\left(\frac{p}{p+v}\right)^{k / 2} H_{k}^{[p+v]}$. Hence, over an additive Gaussian noise channel $g_{v}$, if we perturb the input $g_{p}$ in the direction $H_{k}^{[p]}$ by an amount $\varepsilon$, we will perturb the output $g_{p+v}$ in the direction $H_{k}^{[p+v]}$ by an amount $\left(\frac{p}{p+v}\right)^{k / 2} \varepsilon$. Such a perturbation in $H_{k}$ implies that the output entropy is reduced (compared to not perturbing) by $\left(\frac{p}{p+v}\right)^{k} \frac{\varepsilon^{2}}{2}$ (if $k \geq 3$ ).

\section{A. Hermite Coding: Formalities}

With previous results, we will use the Hermite polynomials as our directions to perturb Gaussians. The Hermite polynomial corresponding to $k=0$ is $H_{0}^{[p]}=1$ and is clearly not a valid direction as it violates (6). But $H_{0}^{[p]}=1$ also implies, from the orthogonality property of the Hermite basis, that $H_{k}^{[p]}$ satisfies (6) for any $k>0$. However, it is only for $k$ even that $H_{k}^{[p]}$ satisfies (5). On the other hand, for any $\delta>0$, we have that $H_{k}^{[p]}+\delta H_{4 k}^{[p]}$ satisfies (5), whether $k$ is even or not. Now, if we consider the direction $-H_{k}^{[p]}$, (5) is not satisfied for both $k$ even and odd. But again, for any $\delta>0$, we have that $-H_{k}^{[p]}+\delta H_{4 k}^{[p]}$ satisfies (5). Hence, in order to ensure (5), we will often work in the proofs with $\pm H_{k}^{[p]}+\delta H_{4 k}^{[p]}$, although it will essentially allow us to reach the performance achieved by any $\pm H_{k}^{[p]}$ (odd or even), since we will then take $\delta$ arbitrarily small and use continuity arguments.

Convention: We will drop the variance upper script in the Hermite terms whenever a Gaussian density with specified variance is perturbed, i.e., the density $g_{p}\left(1+\varepsilon H_{k}\right)$ always denotes $g_{p}\left(1+\varepsilon H_{k}^{[p]}\right)$, no matter what $p$ is. We can afford such a notation since Lemma 2 will always force the Hermite term to have the right variance in our problems. Same treatment is done for $\|\cdot\|_{g_{p}}$ and $\|\cdot\|$.

Now, in order to evaluate the entropy of a perturbation, i.e., $h\left(g_{p}(1+\varepsilon L)\right)$, we can express it as the entropy of $h\left(g_{p}\right)$ minus the divergence gap, as in (7), and then use Lemma 1 for the approximation. But this is correct if $g_{p}(1+\varepsilon L)$ has 
the same first two moments as $g_{p}$. Hence, if $L$ contains only $H_{k}$ 's with $k \geq 3$, the previous argument can be used. But if $L$ contains $H_{1}$ and/or $H_{2}$ terms, the situation can be different. Next Lemma describes this.

Lemma 2: Let $\delta, p>0$ and

$$
b \widetilde{H}_{k}= \begin{cases}b\left(H_{k}+\delta H_{4 k}\right), & \text { if } b \geq 0, \\ b\left(H_{k}-\delta H_{4 k}\right), & \text { if } b<0 .\end{cases}
$$

We have for any $\alpha_{k} \in \mathbb{R}, k \geq 1, \varepsilon>0$

$$
\begin{aligned}
& h\left(g_{p}\left(1+\varepsilon \sum_{k \geq 1} \alpha_{k} \widetilde{H}_{k}\right)\right)= \\
& h\left(g_{p}\right)-D\left(g_{p}\left(1+\varepsilon \sum_{k \geq 1} \alpha_{k} \widetilde{H}_{k}\right) \| g_{p}\right)+\frac{\varepsilon \alpha_{2}}{\sqrt{2}} .
\end{aligned}
$$

Finally, we will need the following lemma.

Lemma 3: We have $g_{a} H_{k}^{[a]} \star g_{b} H_{l}^{[b]}=C g_{a+b} H_{k+l}^{[a+b]}$, where $C$ is a constant depending only on $a, b, k$ and $l$. In particular if $k=l=1$, we have $C=\frac{\sqrt{2 a b}}{a+b}$.

\section{RESUlTS FOR THE IC}

Definition 2: Let

$F_{k}(a, p)=\lim _{\delta \searrow 0} \lim _{\varepsilon \searrow^{0}} \frac{2}{\varepsilon^{2}}\left[S_{a, p}\left(X_{1}, X_{2}\right)-S_{a, p}\left(X_{1}^{G}, X_{2}^{G}\right)\right]$ where $X_{1} \sim g_{p}\left(1+\varepsilon \widetilde{H}_{k}\right), X_{2} \sim g_{p}\left(1-\varepsilon \widetilde{H}_{k}\right)$, with $\widetilde{H}_{k}$ defined in (14), and $X_{1}^{G}, X_{2}^{G} \stackrel{\text { iid }}{\sim} g_{p}$.

That is, $F_{k}(a, p)$ represents the gain (positive or negative) of using $X_{1}$ perturbed along $H_{k}$ and $X_{2}$ perturbed along $-H_{k}$ with respect to using Gaussian distributions.

Theorem 1: We have for $k \geq 2$

$$
F_{k}(a, p)=\left[a^{2}\left(1+p+p a^{2}\right)\right]^{k}-\left(1-a^{k}\right)^{2}\left(1+p a^{2}\right)^{k} .
$$

For any fixed $p$, the function $F_{k}(\cdot, p)$ has a unique positive root, below which it is negative and above which it is positive.

Proposition 3: Treating interference as noise with iid Gaussian inputs does not achieve the sum-capacity of the symmetric IC (synch or asynch) and is outperformed by $X_{1} \sim g_{p}(1+$ $\left.\varepsilon \widetilde{H}_{3}\right)$ and $X_{2} \sim g_{p}\left(1-\varepsilon \widetilde{H}_{3}\right)$, if $F_{3}(a, p)>0$.

Proposition 4: For the symmetric synch IC, when treating interference as noise, time sharing improves on the iid Gaussian distribution if $F_{2}(a, p)>0$.

Definition 3: Blind time sharing over a block length $n$ (assumed to be even) between two users, refers to sending non-zero power symbols only at the instances marked with a 1 in $(1,0,1,0,1,0, \ldots 1,0)$ for the first user, and zero power symbols only at the instances marked with a 1 in $(1,1, \ldots, 1,0,0, \ldots, 0)$ for the second user.

Proposition 5: For the symmetric totally asynch IC, if the receivers only know the asynchronization delay, Gaussian blind time sharing improves on treating interference as noise with iid Gaussian distributions if $B_{2}(a, p)>0$, where $B_{2}(a, p)=\frac{1}{4}\left(\log (1+2 p)+\log \left(1+\frac{2 p}{1+2 a^{2} p}\right)\right)-\log \left(1+\frac{p}{1+a^{2} p}\right)$. If the receivers do not know the asynchronization delay, blind time sharing cannot improve on treating interference as noise with iid Gaussian distributions if $B_{2}(a, p) \leq 0$.
How to read these results: We have four thresholds to keep track of. When treating interference as noise we have:

- $T_{1}(p)$ is when $p a^{3}+a-\frac{1}{2}=0$. If $a \leq T_{1}(p)$, we know from [2], [6], [7] that iid Gaussian inputs are sumcapacity achieving.

- $T_{2}(p)$ is when $F_{2}(a, p)=0$. If $a>T_{2}(p)$, we know from Prop. 4 that, if synchronization is permitted, time sharing improves on iid Gaussian inputs. This regime matches with the so-called moderate regime defined in [3].

- $T_{3}(p)$ is when $F_{3}(a, p)=0$. If $a>T_{3}(p)$, we know from Prop. 3 that non-Gaussian distributions (opposites in $H_{3}$ ) improve on iid Gaussian inputs.

- $T_{4}(p)$ is when $B_{2}(a, p)=0$. If $a>T_{4}(p)$, we know from Prop. 5 that, even if the users are totally asynchronized, blind time sharing improves on iid Gaussian inputs (if the receivers know the delay).

The question is now, how are these thresholds ranked. It turns out that $0<T_{1}(p)<T_{2}(p)<T_{3}(p)<T_{4}(p)$. And if $p=1$, the above inequality reads as $0.424<0.605<0.680<1.031$. This implies the following for a decoder that treats interference as noise. Since $T_{2}(p)<T_{3}(p)$, it is first better to time share than using non-Gaussian distributions along $H_{3}$. But this is useful only if time-sharing is permitted, i.e., for the synch IC. However, for the asynchronized IC, since $T_{3}(p)<T_{4}(p)$, we are better off using the non-Gaussian distributions along $H_{3}$ before a Gaussian input scheme, even with blind timesharing. Finally, we notice that there is still a gap between $T_{1}(p)$ and $T_{2}(p)$, and we cannot say if, in this range, iid Gaussian inputs are still optimal, or if another class of nonGaussian inputs (far away from Gaussians) can outperform them. However, we have noticed something mysterious. In theorem 1 , we asked for $k \geq 2$. Now, we can still wonder what the inequality achieved by requiring the expression of theorem 1 to be positive looks like for $k=1$. And it turns out that this is precisely $p a^{3}+a-\frac{1}{2}>0$, i.e., the complement range delimited by $T_{1}(p)$. But the RHS of theorem 1 for $k=1$ is not equal to $F_{1}(a, p)$, and indeed, it would not make sense that moving along $H_{1}$, which changes the mean with a fixed second moment within Gaussians, would allow us to improve on the iid Gaussian scheme. Yet, getting to the exact same condition, when working on the problem of improving on the iid Gaussian scheme, seems to be a strange coincidence.

\section{Proof of Theorem 1:}

Let $\varepsilon, \delta>0$ and let $X_{1}$ and $X_{2}$ be respectively distributed as $g_{p}\left(1+\varepsilon\left[H_{k}+\delta H_{4 k}\right]\right)$ and $g_{p}\left(1-\varepsilon\left[H_{k}-\delta H_{4 k}\right]\right)$, where $k \neq 1,2$. We have $I\left(X_{1}, X_{1}+a X_{2}+Z_{1}\right)=h\left(X_{1}+a X_{2}+\right.$ $\left.Z_{1}\right)-h\left(a X_{2}+Z_{1}\right)$, where $X_{k}^{G}$ are independent Gaussian 0mean and $p$-variance random variables. Let us first analyze $h\left(X_{1}+a X_{2}+Z_{1}\right)$. The density of $X_{1}+a X_{2}+Z_{1}$ is given by $g_{p}\left(1+\varepsilon\left[H_{k}+\delta H_{4 k}\right]\right) \star g_{a^{2} p}\left(1-\varepsilon\left[H_{k}-\delta H_{4 k}\right]\right) \star g_{1}$ which, from Proposition 2, equals to $g_{p+a^{2} p+1}(1+D)$, where $D$ is 
given by

$\varepsilon\left\{\left[\left(\frac{p}{p+a^{2} p+1}\right)^{\frac{k}{2}} H_{k}+\delta\left(\frac{p}{p+a^{2} p+1}\right)^{2 k} H_{4 k}\right]\right.$
$\left.-\left[\left(\frac{a^{2} p}{p+a^{2} p+1}\right)^{\frac{k}{2}} H_{k}-\delta\left(\frac{a^{2} p}{p+a^{2} p+1}\right)^{2 k} H_{4 k}\right]-\varepsilon L\right\}$

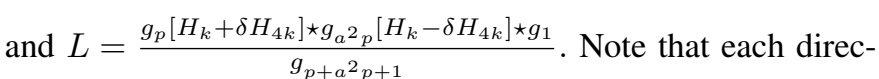
tion in each line of the $\{\cdot\}$ bracket above, including $L$, satisfy (5) and (6). Using Lemma 3, we have

$$
\begin{aligned}
& L=\frac{g_{p}\left[H_{k}+\delta H_{4 k}\right] \star g_{a^{2} p+1}\left[\left(\frac{a^{2} p}{a^{2} p+1}\right)^{\frac{k}{2}} H_{k}-\left(\frac{a^{2} p}{a^{2} p+1}\right)^{2 k} \delta H_{4 k}\right.}{g_{p+a^{2} p+1}} \\
& =C_{1} H_{2 k}^{\left[p+a^{2} p+1\right]}+C_{2} H_{5 k}^{\left[p+a^{2} p+1\right]}+C_{3} H_{8 k}^{\left[p+a^{2} p+1\right]}
\end{aligned}
$$

where $C_{1}, C_{2}, C_{3}$ are constants. Therefore, the density of $X_{1}+$ $a X_{2}+Z_{1}$ is a Gaussian $g_{p+a^{2} p+1}$ perturbed along the direction $H_{k}$ in the order $\varepsilon$ and several $H_{l}$ with $l \geq 2 k$ in the order $\varepsilon^{2}$ (and other directions but that have a $\delta$ order). So we can use Lemma 2 and write $h\left(X_{1}+a X_{2}+Z_{1}\right)=h\left(X_{1}^{G}+a X_{2}^{G}+\right.$ $\left.Z_{1}\right)-D\left(X_{1}+a X_{2}+Z_{1} \| X_{1}^{G}+a X_{2}^{G}+Z_{1}\right)$ and using Lemma 1 , we have

$$
\begin{aligned}
& D\left(X_{1}+a X_{2}+Z_{1} \| X_{1}^{G}+a X_{2}^{G}+Z_{1}\right) \\
& \doteq \frac{\varepsilon^{2}}{2} \|\left[\left(\frac{p}{p+a^{2} p+1}\right)^{\frac{k}{2}} H_{k}+\delta\left(\frac{p}{p+a^{2} p+1}\right)^{2 k} H_{4 k}\right] \\
& -\left[\left(\frac{a^{2} p}{p+a^{2} p+1}\right)^{\frac{k}{2}} H_{k}-\delta\left(\frac{a^{2} p}{p+a^{2} p+1}\right)^{2 k} H_{4 k}\right] \|^{2} \\
& =\frac{\varepsilon^{2}}{2}\left(1-a^{k}\right)^{2}\left(\frac{p}{p+a^{2} p+1}\right)^{k}+\frac{\varepsilon^{2}}{2} o(\delta) .
\end{aligned}
$$

Hence $h\left(X_{1}+a X_{2}+Z_{1}\right)=h\left(X_{1}^{G}+a X_{2}^{G}+Z_{1}\right)-\frac{\varepsilon^{2}}{2}(1-$ $\left.a^{k}\right)^{2}\left(\frac{p}{p+a^{2} p+1}\right)^{k}+\frac{\varepsilon^{2}}{2} o(\delta)$. More directly, we get $D\left(a X_{2}+\right.$ $\left.Z_{1} \| a X_{2}^{G}+Z_{1}\right) \doteq \frac{\varepsilon^{2}}{2}\left(\frac{a^{2} p}{a^{2} p+1}\right)^{k}+\frac{\varepsilon^{2}}{2} o(\delta)$ and

$I\left(X_{1}, X_{1}+a X_{2}+Z_{1}\right) \doteq I\left(X_{1}^{G}, X_{1}^{G}+a X_{2}^{G}+Z_{1}\right)$

$+\frac{\varepsilon^{2}}{2}\left[\left(\frac{a^{2} p}{a^{2} p+1}\right)^{k}-\left(1-a^{k}\right)^{2}\left(\frac{p}{p+a^{2} p+1}\right)^{k}\right]+\frac{\varepsilon^{2}}{2} o(\delta)$

Finally, one can show that $I\left(X_{2}, X_{2}+a X_{1}+Z_{2}\right)=$ $I\left(X_{1}, X_{1}+a X_{2}+Z_{1}\right)$ and

$$
\begin{aligned}
& I\left(X_{1}, X_{1}+a X_{2}+Z_{1}\right)+I\left(X_{2}, X_{2}+a X_{1}+Z_{2}\right) \\
& \stackrel{\circ}{=} I\left(X_{1}^{G}, X_{1}^{G}+a X_{2}^{G}+Z_{1}\right)+I\left(X_{2}^{G}, X_{2}^{G}+a X_{1}^{G}+Z_{2}\right) \\
& +\varepsilon^{2}\left[\left(\frac{a^{2} p}{a^{2} p+1}\right)^{k}-\left(1-a^{k}\right)^{2}\left(\frac{p}{p+a^{2} p+1}\right)^{k}\right]+\varepsilon^{2} o(\delta) .
\end{aligned}
$$

Hence, if for some $k \neq 3$ we have

$$
\left(\frac{a^{2}}{a^{2} p+1}\right)^{k}-\frac{\left(a^{k}-1\right)^{2}}{\left(p+a^{2} p+1\right)^{k}}>0
$$

we can improve on the iid Gaussian distributions $g_{p}$ by using the respective Hermite perturbations. Now, we could have started with $X_{1}$ and $X_{2}$ distributed as $g_{p}\left(1+\varepsilon b_{k} \widetilde{H}_{k}\right)$ and $g_{p}\left(1+\varepsilon c_{k} \widetilde{H}_{k}\right)$. But one can show that the optimal choice is $b_{k}=-c_{k}$. Moreover, note that for this distribution of $X_{1}$ and $X_{2}$, we could have actually chosen $k=2$ as well. Because, even if Lemma 2 tells us that we must use correction terms, these correction terms will cancel out when we consider the sum-rate, since $b_{k}=-c_{k}$ and since the correction is in $\varepsilon$. There is however another problem with using $k=2$, which is that $g_{p}\left(1+\varepsilon H_{2}\right)$ has a larger second moment than $p$. However, if we use a scheme of block length 2 , we can compensate this excess on the first channel use with the second channel use, and because of the symmetry, we can achieve the desired rate. But this is allowed only with synchronization. Finally, if we work with $k=1$, the proof sees the following modification. In (15), we now have a term in $\mathrm{H}_{2}$. However, even if this term is in the order $\varepsilon^{2}$, we can no longer neglect it, since from Lemma 2, a $\varepsilon^{2} H_{2}$ term in the direction comes out as a $\frac{\varepsilon^{2}}{\sqrt{2}}$ term in the entropy. Hence, we do not get the above condition for $k=1$, but the one obtained by replacing $\left(a^{k}-1\right)^{2}$ with $\left(a^{2}+1\right)$, and the condition for positivity can never be fulfilled.

\section{A. Discussion}

We have used encoders drawn from non-Gaussian distributions along Hermite polynomials as introduced in [1]. If the performance of non-Gaussian inputs is usually hard to analyze, we showed how the neighborhoods of Gaussian inputs can be analyzed in close forms by using the Hermite coordinate system. We found in this paper that using non-Gaussian input distributions (along $\mathrm{H}_{3}$ ) can strictly improve on the Gaussian distribution for the asynch IC, when treating interference as noise. We also recovered the threshold of the moderate regime by using $\mathrm{H}_{2}$ perturbations in the synch setting, showing that this global threshold is reflected in our local setting. We also met mysteriously in our local setting the other global threshold found in [2], [6], [7], below which treating interference as noise with iid Gaussian inputs is optimal. We hope to understand this better with the work in progress. It would be interesting to compare the results obtained with the local analysis and the global ones. The fact that we have observed global results locally, as mentioned previously, gives hope for possible local to global extensions. In a current work in progress, we extend the "Hermite eigenfunction theorem" presented in this paper to a more general result which can apply to problems in the global (non-local) setting.

\section{ACKNOWLEDGMENT}

The first author would like to thank Emre Telatar for his careful reading and his important comments.

\section{REFERENCES}

[1] E. Abbe and L. Zheng, "Non-Gaussian Coding Along Hermite Polynomials for Fading Broadcast Gaussian Channel", accepted to ISIT 2009.

[2] V.S. Annapureddy and V.V. Veeravalli, "Gaussian Interference Network: sum Capacity in the Low Interference Regime and New Outer Bounds on the Capacity Region", Submitted IEEE Trans. Inform. Theory, 2008. 
[3] M. Costa, "On the Gaussian interference channel," IEEE Trans. Inform. Theory, vol. IT-31, pp. 607-615, Sept. 1985.

[4] R. Courant and D. Hilbert, "Methods of Mathematical Physics", Volume I, Wiley-Interscience, 1953.

[5] T. M. Cover and J. A. Thomas, "Elements of Information Theory", John Wiley \& Sons, New York, NY, 1991.

[6] A. S. Motahari, A. K. Khandani, "Capacity Bounds for the Gaussian Interference Channel", CoRR abs/0801.1306: (2008).

[7] X. Shang, G. Kramer, B. Chen, "Outer bound and noisy-interference sumrate capacity for symmetric Gaussian interference channels", CISS 2008: 385-389. 